Pacific Journal of Mathematic 


\title{
CENTRALIZERS OF ABELIAN, NORMAL SUBGROUPS OF HYPERCYCLIC GROUPS
}

\author{
ULRICH SCHOENWAELDER
}

\begin{abstract}
J. L. Alperin proved the following theorem about finite $p$-groups $G$ : if $E$ is maximal among the abelian, normal subgroups of $G$ of exponent dividing $p^{n}$, then $\Omega_{n} \S_{G}(E)=E$, provided that $p^{n} \neq 2$. It turns out that the restriction to $p$-groups and also to finite groups in Alperin's proof is not essential. In fact a similar theorem holds in a large class of hypercyclic groups (Theorem 2.2). By the same method also a modified version (Theorem 2.8) will be obtained, the word "normal" in the assumptions about $E$ being replaced by "characteristic", here $G$ is supposed to be hypercentral; the modification results in enlarging $E$ to a characteristic subgroup $\mathfrak{X}_{G}(E)$ of class 2 in a very definite way before taking its centralizer.
\end{abstract}

The proofs of both theorems rely on a fairly general version (not used in its full strength) of the lemma used by Alperin on $p$-automorphisms of abelian $p$-groups that centralize all elements of order $p$. The first paragraph is devoted to this generalization (Theorem 1.11) and may be of independent interest.

TERminology. We denote by $f$ the set of all functions from the set of all primes to the set of all rational integers extended by the symbol $\infty$. Addition and subtraction are defined on $f$ by $(f \pm q)(p)=$ $f(p) \pm g(p)$, where $\infty$ is handled in the usual manner; also $f \leqq g$ for $f, g \in f$ if and only if $f(p) \leqq g(p)$ for all primes $p$. A function $f \in f$ is called finite, if $f(p)<\infty$ for every prime $p$. The constant functions in $f$ will be denoted by their single value. The function $d \in f$ which is 2 at 2 and 1 elsewhere will play a particular role in our discussion.

Let $f$ be a function in $f$. The nonegative part $f^{+}$of $f$ is defined by $f^{+}(p)=f(p)$ if $f(p) \geqq 0$, and $f^{+}(p)=0$, if $f(p) \leqq 0$. With every torsion element $x$ of a group $X$ there is associated a function $e_{x} \in f$ such that $\Pi\left\{p^{e} x^{(p)} \mid p\right.$ prime $\}$ is the order of $x$. We say that an element $x$ is restrained by $f$, if $x$ is a torsion element and $e_{x} \leqq f^{+}$. The elements of $X$ restrained by $f$ generate a (characteristic) subgroup $\Omega_{f}(X)$ of $X$. We say that $X$ is restrained by $f$, if every element of $X$ is restrained by $f$.

For every torsion element $x$ of a group $X$ and every prime $p$ there is a uniquely determined power $x_{p}$ of $x$ such that the order of $x_{p}$ is a power of $p$ and the order of $x_{p^{\prime}}=x_{p}^{-1} x$ is prime to $p . X_{p}$ is the set of all $p$-elements of $X$. 
A group $G$ is called hypercyclic, if every epimorphic image, not 1 , of $G$ has a cyclic, normal subgroup, not 1 . This implies that every normal subgroup, not 1 , of an epimorphic image $H$ of $G$ contains a cyclic, normal subgroup, not 1 , of $H$. A group $G$ is called hypercentral, if every epimorphic image, not 1 , of $G$ has a nontrivial center. This implies that every normal subgroup, not 1 , of an epimorphic image $H$ of $G$ intersects the center of $H$ nontrivially.

We use the notation $(a, b)=a \circ b=a^{-1} a^{b}$ where $b$ is an endomophism or a group element and $a^{b}=b^{-1} a b$. If $b$ operates on $A$, then $A \circ b$ is the set of all $(a, b)$ with $a \in A . \quad(a, b, c)=((a, b), c)$. An element or automorphism $x$ of $X$ centralizes the $x$-invariant factor $B / A$, if $x$ fixes every element in $B / A . \mathfrak{N}_{X}(A)=$ normalizer of $A$ in $X, \mathfrak{\Im}_{X}(A)=$ centralizer of $A$ in $X, 3(X)=$ center of $X, X^{\prime}=$ commutator subgroup of $X, A_{X}=$ largest normal subgroup of $X$ contained in $A,\langle S\rangle=$ subgroup generated by the set $S, p^{\prime}=$ set of all primes different from $p$.

1. After a few lemmas of a general nature this paragraph will be concerned with torsion automorphisms that centralize $\Omega_{d}(G)$.

Lemma 1.1. For a group $G$, an endomorphism $x$ of $G$ and an element $b$ of $G$ define the elements $b_{i}, i \geqq 0, b y$

$$
b_{0}=b, b_{i}^{x}=b_{i} b_{i+1},
$$

and assume $b_{i} b_{i+1}=b_{i+1} b_{i}$ for $i>1$. Then for every integer $s \geqq 0$,

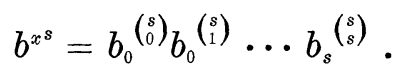

Proof. Since $\left(\begin{array}{l}s \\ 0\end{array}\right)=1$ for all $s>0$, the statement is true for $s=0$. We proceed by induction on $s$ to get

$$
\begin{aligned}
& b^{x^{s+1}}=\left[b_{0}^{\left(\begin{array}{c}
s \\
0
\end{array}\right)}\right]^{x}\left[b_{1}^{\left(\begin{array}{l}
s \\
1
\end{array}\right)}\right]^{x} \cdots\left[b_{s}^{\left(\begin{array}{c}
s \\
s
\end{array}\right)}\right]^{x} \\
& =\left[b_{0} b_{1}\right]^{\left(\begin{array}{l}
s \\
0
\end{array}\right)}\left[b_{1} b_{2}\right]^{\left(\begin{array}{l}
s \\
1
\end{array}\right)} \cdots\left[b_{s} b_{s+1}\right]^{\left(\begin{array}{c}
s \\
s
\end{array}\right)}
\end{aligned}
$$

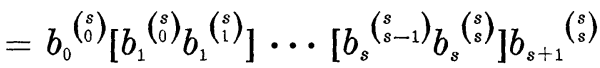

$$
\begin{aligned}
& =b_{1}{ }^{\left(\begin{array}{c}
s+1 \\
0
\end{array}\right)} b_{1}{ }^{\left(\begin{array}{c}
s+1 \\
1
\end{array}\right)} \cdots b_{s}^{\left(\begin{array}{c}
s+1 \\
s
\end{array}\right)} b_{s+1}^{\left(\begin{array}{c}
s+1 \\
s+1
\end{array}\right)}
\end{aligned}
$$

where we use the formulas $\left(\begin{array}{l}s \\ i\end{array}\right)+\left(\begin{array}{c}s \\ i+1\end{array}\right)=\left(\begin{array}{l}s+1 \\ i+1\end{array}\right)$ and $\left(\begin{array}{l}s \\ 0\end{array}\right)=\left(\begin{array}{l}s \\ s\end{array}\right)=$ 1. This proves the lemma.

LEMMA 1.2. If $x$ is an automorphism of the group $G$ that centralizes the subgroup $U$ of $G$, then $U$ and $\left\langle\mathfrak{R}_{G}(U) \circ x\right\rangle$ centralize each other. 
Proof. Let $u \in U$ and $g \in \mathfrak{R}_{G}(U)$. Then $u^{g} \in U$, hence $u^{g}=\left(u^{g}\right)^{x}=$ $\left(u^{x}\right)^{g^{x}}=u^{g^{x}}$. Therefore $g^{-1} g^{x}$ centralizes $U$ which proves the lemma.

LEMmA 1.3. Let $U$ be a subgroup of a group $G$ and $x$ an automorphism of $G$ that centralizes $U$ and satisfies $\langle G \circ x\rangle \leqq U$. Then

(1) $\langle G \circ x\rangle \leqq \mathfrak{E}_{U}\left(U_{G}\right)$;

(2) if the set $G \circ x$ has finite exponent $n$, then $x$ is a torsion automorphism of order dividing $n$;

(3) if $x$ is a torsion automorphism, then for every $g \in G$ the order of $g \circ x$ divides the order of $x$.

Proof. (1) follows from Lemma 1.2 applied to $U_{G}$. Let $g \in G$ and put $h=g^{-1} g^{x} \in G \circ x \subseteq U$. Then $g^{x}=g h$ and, by induction, $g^{x^{r}}=g h^{r}$. In particular, under the assumption of (2), $g^{x^{n}}=g h^{n}=g$. Hence $x^{n}=1$ proving (2). On the other hand, if $x$ is a torsion automorphism of order $m$, then $g=g^{x^{m}}=g h^{m}$, hence $h^{m}=1$ proving (3).

Lemma 1.4. [4, p. 49, 1.5 Hilfssatz.] Every automorphism of a finite p-group $P$ that centralizes $\Omega_{d}(P)$ has order a power of $p$.

Proof. Let $P$ be a counterexample of minimal order and $x \neq 1$ a $p^{\prime}$-automorphism of $P$ centralizing $\Omega_{d}(P)$. Assume by way of contradiction that $\langle P \circ x\rangle$ is a proper subgroup of $P$. $P$ being a minimal counterexample and $\langle P \circ x\rangle$ being $x$-admissible, $\langle P \circ x\rangle$ must be centralized by $x$. So Lemma 1.3 (2) implies $x=1$, a contradiction. Therefore $P=\langle P \circ x\rangle$. Since $P$ is solvable, $P^{\prime}$ is a proper, $x$-admissible subgroup of $P$. Hence $P^{\prime}$ is centralized by $x$. By Lemma 1.2, $P^{\prime} \cong \complement_{P}(\langle P \circ x\rangle)=$ $3(P)$ and $P$ has class 2. Let $t$ be minimal such that $P=\Omega_{t+1}(P)$, hence $t \geqq 1$, and let $g \in P$ be an element of order dividing $p^{t+1}$. Then $g^{p^{t}} \in \Omega_{1}(P)$ is centralized by $x$, hence

$$
\left(g^{1} g^{x}\right)^{p^{t}}=\left(g^{-1}\right)^{p^{t}}\left(g^{x}\right)^{p^{t}}\left(g^{x}, g^{-1}\right)^{\left(p^{t}\right)}=\left(g^{x}, g^{-1}\right)^{\left(p_{2}^{t}\right)},
$$

see [9, p. 8., (10)]. If $p \neq 2$, then $p^{t}$ divides $\left(\begin{array}{c}p^{t} \\ 2\end{array}\right)$; put $s=t$. If $p=2$, then $p^{t-1}$ divides $\left(\begin{array}{l}p^{t} \\ 2\end{array}\right)$; put $s=t-1$. Now

$$
\left(g^{x}, g^{-1}\right)^{p^{s}}=\left(g^{x}, g^{-p^{s}}\right),
$$

see $\left[9\right.$, p. 80, (9)], and $g^{p^{s}} \in \Omega_{d}(P) \subseteq 3(P)$ by Lemma 1.2. Therefore

$$
\left.\left(g^{x}, g^{-p^{s}}\right)=1,\left(g^{x}, g^{-1}\right)^{\left(p^{p} t\right.}\right)=1, \quad \text { and } \quad\left(g^{-1} g^{x}\right)^{p^{t}}=1
$$

showing that

$$
P=\langle P \circ x\rangle=\left\langle\Omega_{t+1}(P) \circ x\right\rangle \sqsubseteq \Omega_{t}(P) \subset P,
$$


a contradiction. No counterexamples exist.

CoROLlaRY 1.5. Let $E$ be a subgroup of a finite group $G$ such that $\mathfrak{S}_{G}(E)$ has a p-Sylow subgroup $S$ which satisfies $\Omega_{d}(S) \cong E$. Then $\mathfrak{夭}_{G}(E)$ has a normal p-complement.

Proof. Suppose $U$ is a subgroup of $S$ and $x$ is a $p^{\prime}$-element of $\Re_{C}(U), C=\mathfrak{S}_{G}(E)$. Then $\Omega_{d}(U) \subseteq \Omega_{d}(S) \subseteq E$ is centralized by $x$. Lemma 1.4 implies that $U$ is centralized by $x$. By a well-known theorem of Frobenius [3], $C$ has a normal $p$-complement.

Proposition 1.6. If $A$ is a locally finite, normal subgroup of the group $B$, then any torsion automorphism of $B$ that leaves $A$ invariant and centralizes $B / A$ and $\Omega_{d}(A)$ has order divisible by primes that are orders of elements in $A$ only.

Proof. Such an automorphism $x$ is the product of its primary components $x_{q}, q$ prime, $x_{q}$ being a power of $x$. Put $y=x_{q}$ and assume that $q$ is not the order of an element in $A$. Pick $a \in A$. Being finitely generated $F=\left\langle a^{\langle y\rangle}\right\rangle$ is a finite, $y$-admissible subgroup of $A$. For any prime $p$ the number of $p$-Sylow subgroups of $F$ is prime to $q$ and $y$ normalizes at least one $p$-Sylow subgroup $P$ of $F$. By Lemma 1.4, $P$ is centralized by $y$. So $F=\langle P| p$ prime $\rangle$ is centralized by $y$ and, in particular, $a$ is centralized by $y$.

Pick $b \in B . \quad A$ being a torsion group, $b^{-1} b^{y} \in A$ has finite order prime to $q$. By Lemma 1.3 (3) this order divides the order of $y$, $b^{-1} b^{y}=1$ and $x_{q}=y=1$.

We shall only need the following special case of Proposition 1.6.

CoRollary 1.7. Let $A$ be a normal torsion subgroup of a group $B$, assume $\Omega_{i}(A) / \Omega_{i-d}(A) \subseteq 3\left(\Omega_{i+1}(A) / \Omega_{i-d}(A)\right)$ for all functions $i \in \mathcal{f}$ with $i \geqq d$, let $x$ be a torsion automorphism of $B$ that leaves $A$ invariant and centralizes $B / A$ and $\Omega_{d}(A)$. If the order of $x$ is prime to the order of every element in $A$, then $x=1$.

Proof. $A$ is locally finite, since it is the union of solvable (hence locally finite) torsion subgroups. Exploiting the structure of $A$ one may prove Corollary 1.7 also without reference to Lemma 1.4.

REMARK 1.8. The quaternion group of order 8 shows that $\Omega_{d}(P)$ in Lemma 1.4 may not be replaced by $\Omega_{1}(P)$. However, if $P$ is abelian, this may be done. Similarly in Proposition 1.6 and Corollary $1.7 \Omega_{d}(A)$ may be replaced by $\Omega_{1}(A)$ provided that finite 2 -subgroups of $A$ are abelian. 
LEMMA 1.9. Let $A$ be a torsion group with abelian factors $\Omega_{i}(A) / \Omega_{i-1}(A)$ for all finite functions $i \in f_{\text {. }}$ Let $0 \leqq k \in \mathcal{f}$ satisfy $k(2) \geqq 2$ and $\Omega_{k}(A) \cong 3(A)$, assume that only finitely many primes are orders of elements in $A$. Then any automorphism of $A$ that centralizes $\Omega_{k}(A)$ also centralizes all the factors $\Omega_{n}(A) / \Omega_{n-k}(A)$ for finite functions $n \in f$.

Proof. Clearly by the structure of $A, \Omega_{r}(A)$ is ' restrained by $r$ for $r \in \mathcal{f}$. If there are counterexamples, then there are also counterexamples of finite exponent, since only finitely many primes are orders of elements in $A$. Let $A$ be a counterexample of minimal (finite) exponent $\Pi p^{m(p)}$. Then automorphisms of $A$ that centralize $\Omega_{k}(A)$ also centralize

$$
\Omega_{j}(A) / \Omega_{j-k}(A)
$$

for $j<m$. In particular, $A$ centralizes $\Omega_{j}(A) / \Omega_{j-k}(A)$ for $j<m$.

Let $x$ be an automorphism of $A$ that centralizes $\Omega_{k}(A)$, pick

$$
a \in \Omega_{m}(A)=A \text {, }
$$

and let $p$, a prime, be the order of an element in $A$. Let $g_{t}$ have the value $t$ at $p$ and 0 elsewhere. Then $\left(a^{x}, a^{-1}\right)$ lies in $\Omega_{m-g_{1}}(A)$, since $\Omega_{m}(A) / \Omega_{m-g_{1}}(A)$ is abelian, and this commutator commutes with $a^{x}$ and $a^{-1} \bmod \Omega_{m-g_{1}-k}(A)$, since $A$ centralizes $\Omega_{m-g_{1}}(A) / \Omega_{m-g_{1}-k}(A)$. Therefore

$$
\left(a^{-1} a^{x}\right)^{p^{t}} \equiv\left(a^{-1}\right)^{p^{t}}\left(a^{x}\right)^{p^{t}}\left(a^{x}, a^{-1}\right)^{\left(p_{2}^{t}\right)} \bmod \Omega_{m-g_{1}-k}(A)
$$

for natural numbers $t$, ef. [9, p.81, (10)]. Since $x$ centralizes

$$
\Omega_{m-g_{1}}(A) / \Omega_{m-g_{1}-k}(A)
$$

by the minimality of the exponent of $A$, we have

$$
\left(a^{-1}\right)^{p^{t}}\left(a^{x}\right)^{p^{t}}=\left(a^{p^{t}}\right)^{-1}\left(a^{p^{t}}\right)^{x} \equiv 1 \bmod \Omega_{m-g_{1}-k}(A)
$$

and

$$
\left(a^{-1} a^{x}\right)^{p^{t}} \equiv\left(a^{x}, a^{-1}\right)^{\left(p_{2}^{t}\right)} \bmod \Omega_{m-g_{1}-k}(A) .
$$

Using a well-known formula, cf. [9, p. 80, (9)], and remembering that $A$ centralizes $\Omega_{m-g_{1}}(A) / \Omega_{m-g_{1}-k}(A)$ we get

$$
\left(a^{x}, a^{-1}\right)^{p} \equiv\left(\left(a^{x}\right)^{p}, a^{-1}\right) \equiv 1 \bmod \Omega_{m-g_{1}-k}(A) .
$$

Assume first that $p \neq 2$ and choose $t=1$. Then $p$ divides $\left(\begin{array}{l}p \\ 2\end{array}\right)$. Hence by (1) and (2), $\left(a^{-1} a^{x}\right)^{p} \equiv 1 \bmod \Omega_{m-g_{1}-k}(A), a^{-1} a^{x} \in \Omega_{m-k}(A)$, and $\dot{x}$ centralizes $\Omega_{m}(A) / \Omega_{m-k}(A)$.

If $p=2$, choose $t=2$. Then $p$ divides $\left(\begin{array}{l}p^{t} \\ 2\end{array}\right)$ and it follows (2) that $\left(a^{x}, a^{-1}\right)^{\left(\begin{array}{c}p^{t} \\ 2\end{array}\right)} \equiv 1 \bmod \Omega_{m-g_{1}-k}(A)$. So (1) implies

$$
\left(a^{-1} a^{x}\right)^{p^{2}} \equiv 1 \bmod \Omega_{m-g_{1}-k}(A)
$$


and $\quad a^{-1} a^{x} \in \Omega_{m-g_{1}-k+g_{2}}(A) \leqq \Omega_{m-g_{1}}(A)$, since $k(2) \geqq 2 \quad$ implies $k \geqq g_{2}$. Therefore

$$
\left(a^{x}, a^{-1}\right)=\left(a\left(a^{-1} a^{x}\right), a^{-1}\right)=\left(a^{-1} a^{x}, a^{-1}\right)
$$

lies in $\Omega_{m-g_{1}-k}(A)$, and $t=1$ in (1) implies

$$
\left(a^{-1} a^{x}\right)^{p} \equiv 1 \bmod \Omega_{m-g_{1}-k}(A) .
$$

Consequently $a^{-1} a^{x} \in \Omega_{m-k}(A)$, and $x$ centralizes $\Omega_{m}(A) / \Omega_{m-k}(A)$.

Lemma 1.10. Let $0 \leqq k \in f$ satisfy $k(2) \geqq 2$, let $A$ be a torsion group with $\Omega_{i}(A) / \Omega_{i-k}(A) \cong 3\left(\Omega_{i+1}(A) / \Omega_{i-k}(A)\right)$ for all finite functions $i \in f$, and assume that only finitely many primes are orders of elements in $A$. Then any automorphism of $A$ that centralizes $\Omega_{k}(A)$ also centralizes all the factors $\Omega_{n}(A) / \Omega_{n-k}(A)$ for finite functions $n \in f$.

Proof. Let $A$ be a counterexample of minimal (finite) exponent $\Pi p^{m(p)}$. Then $\Omega_{k}(A) \neq 1$ and there exists a prime $p$ with $k(p)>0$ and $\Omega_{g}(A) \neq 1$ where $g(p)=1$ and 0 elsewhere. By assumption $\bar{A}=$ $\Omega_{m}(A) / \Omega_{m-g-k}(A)$ satisfies $\Omega_{k}(\bar{A}) \subseteq 3(\bar{A})$, since $\Omega_{k}(\bar{A})=\Omega_{m-g}(A) / \Omega_{m-g-k}(A)$. By the minimality of the exponent of $A$, an automorphism $x$ of $A$ that centralizes $\Omega_{k}(A)$ also centralizes $\Omega_{m-g}(A) / \Omega_{m-g-k}(A)=\Omega_{k}(\bar{A})$. Therefore by Lemma $1.9, x$ centralizes $\Omega_{k+y}(\bar{A}) / \Omega_{g}(\bar{A})$, i.e., $\Omega_{m}(A) / \Omega_{m-k}(A)$.

THEOREM 1.11. Let $A$ be a normal torsion subgroup of a group $B$, assume $\Omega_{i}(A) / \Omega_{i-d}(A) \subseteq 3\left(\Omega_{i+1}(A) / \Omega_{i-d}(A)\right)$ for all finite functions $i \in f$, let $x$ be a torsion automorphism of $B$ that leaves $A$ invariant and centralizes $B / A$ and $\Omega_{d}(A)$, and let $f \geqq 0$ be a function in $f$. Then $x$ centralizes $B / \Omega_{f}(A)$, if and only if $x$ is restrained by $f$.

Proof. (1) To prove the if-part of the theorem we shall assume without loss of generality that $f$ is finite and assumes only finitely many positive values, because $x$ is a torsion automorphism.

Assume first that the statement in question is false for some group $A$ of finite exponent. Then there are counterexamples $A$ of minimal finite exponent $\Pi p^{j(p)}$ of $A$. Choose one where also $f$ is minimal with respect to the partial ordering $\leqq$. It follows $j \neq 0, f \neq 0$, and $x \neq 1$. There exists a prime $q$ such that both $A^{q} \neq 1$ and $q$ divides the order of $x$, since otherwise Corollary 1.7 would imply $x=1$. Define $g \in f$ to be 1 at $q$ and 0 elsewhere. $A / \Omega_{g}(A)$ is restrained by $j-g<j$ and has the required structure. $x$ induces in $B / \Omega_{g}(A)$ an automorphism that leaves $A / \Omega_{g}(A)$ invariant and centralizes $\left[B / \Omega_{g}(A)\right] /\left[A / \Omega_{g}(A)\right]$ and $\Omega_{d}\left(A / \Omega_{g}(A)\right)$; this last fact follows from Lemma 1.10. So the minimality of $j$ yields that $x$ centralizes $\left[B / \Omega_{g}(A)\right] / \Omega_{f}\left(A / \Omega_{q}(A)\right)$, i.e., $B / \Omega_{f+g}(A)$.

Again by our hypothesis and Lemma $1.10, x$ centralizes 


$$
\Omega_{f}(A) / \Omega_{f-d}(A) \text { and } \Omega_{f+g}(A) / \Omega_{f+g-d}(A) .
$$

So we may apply Lemma 1.1 for any $b \in B$ to get

$$
b^{x q} \equiv b b_{1}^{q} b_{2}^{\left(\begin{array}{c}
q \\
2
\end{array}\right)} \bmod \Omega_{f-d}(A)
$$

with $b_{1} \in \Omega_{f+g}(A), b_{2} \in \Omega_{f+g-d}(A) \subseteq \Omega_{f}(A), b_{3} \in \Omega_{f-d}(A)$, since

$$
b_{1} b_{2} \equiv b_{2} b_{1} \bmod \Omega_{f-d}(A)
$$

by the structure of $A$. If $q \neq 2$, then $b_{2}{ }^{\left(\begin{array}{c}q \\ 2\end{array}\right)} \in \Omega_{f-g}(A)$. If $q=2$, then even $b_{2} \in \Omega_{f \rightarrow g}(A)$. Hence

$$
b^{x q} \equiv b b_{1}^{q} \bmod \Omega_{f-g}(A) .
$$

On the other hand by the minimality of $f, x^{q}$ being restrained by $f-g \geqq 0$ centralizes $B / \Omega_{f-g}(A)$ so that

$$
b \equiv b^{x^{q}} \bmod \Omega_{f-g}(A) .
$$

Hence $b_{\llcorner}^{q} \in \Omega_{f \rightarrow g}(A)$ and $b_{1} \in \Omega_{f}(A)$. This signifies that $x$ centralizes $B / \Omega_{f}(A)$ contradicting our assumption and proving the statement for groups $A$ of finite exponent.

Now consider the general case and let $b \in B$. Since $A$ is a torsion group, there exists a function $j \geqq f$ in $f$ such that $\Omega_{j}(A)$ has finite exponent and contains $b^{-1} b^{x}$. Hence $x$ leaving $\Omega_{j}(A)$ invariant centralizes $\langle b\rangle \Omega_{j}(A) / \Omega_{j}(A)$. By what we have already proved above we may conclude that $x$ centralizes $\langle b\rangle \Omega_{j}(A) / \Omega_{f}(A)$, whence $b^{x} \equiv b \bmod \Omega_{f}(A)$. This shows that $x$ centralizes $B / \Omega_{f}(A)$.

(2) Conversely, if $x$ is a torsion automorphism of $B$ centralizing $B / \Omega_{f}(A)$, we may assume $x \neq 1$. Let $p$ be a prime that divides the order of $x$ and define $g \in \mathcal{f}$ to be 1 at $p$ and 0 elsewhere. There exists $h_{b} \leqq f$ depending on $b \in B$ such that $\Omega_{h_{b}}(A)$ has finite exponent and contains $b_{1}=b^{-1} b^{x}$. By Lemma 1.10 applied to $\Omega_{h_{b}}(A), b_{2}=b_{1}^{-1} b_{1}^{x}$ is contained in $\Omega_{h_{b}-d}(A) \subseteq \Omega_{f-g}(A)$. Therefore

$$
b^{x^{p}} \equiv b b_{1}^{p} \bmod \Omega_{f-g}(A)
$$

by Lemma 1.1. But $b_{1}^{p} \in \Omega_{f-g}(A)$, hence $x^{p}$ centralizes $B / \Omega_{f-g}(A)$.

By induction on the order of $x, x^{p}$ is restrained by $f-g$. So $x$ is restrained by $f$.

REMARK 1.12. H. Leptin [6, p. 101] proved that in the case of a reduced abelian $p$-group $A$ with $p \geqq 5$ the conclusion of Theorem 1.11 remains valid under the weaker hypothesis that $x$ only centralizes certain factors of $\Omega_{1}(A)$ instead of $\Omega_{1}(A)$ as a whole.

REMARK 1.13. Let $A$ be an abelian 2-group of exponent $\geqq 8$ and 
let $x$ be the automorphism of $A$ that maps every element onto its inverse. Then $x$ centralizes $\Omega_{1}(A)$ and has order 2 , but does not centralize $A / \Omega_{1}(A)$.

2. Let $E$ be a normal subgroup of the $p$-group $G$ and denote by $\mathfrak{S}^{k}(E)$ the subgroup of $G$ formed by all the elements of $G$ that centralize all the factors $\Omega_{i}(E) / \Omega_{i-k}(E), i \geqq k$. The following proposition may be generalized to the case where $E$ satisfies $E \subseteq \varsigma^{k}(E)$ instead of being abelian $(k=\infty)$. But if $x \in \Im^{k}(E)$ satisfies $x^{p} \in E$ and $(x, g) \subseteq E$ then it does not follow that the subgroup $W$ generated by $E$ and $x$ satisfies $W \subseteq \varsigma^{k}(W)$, since $\Omega_{1}(W) \subseteq 3(W)$ may be violated. Hence no application of the proposition in this case which would be similar to the proof of Theorem 2.2 or Theorem 2.8 below is to be expected. Consequently we shall restrict our attention to the abelian case and follow Alperin's argument.

Proposition 2.1. Let $G$ be a group, $E$ an abelian subgroup of $G, E_{1}$ a subgroup of $G$ that contains $E$, and $f \geqq 0$ a function in $f$ such that

(1) $f(2) \neq 1$,

(2) if $h$ is a function in $f$ with $0 \leqq h \leqq f$ and if $\Omega_{h} \Im_{G}\left(E_{1}\right)$ is restrained by $f$, then $\Omega_{h} \complement_{G}\left(E_{1}\right) \subseteq E$,

(3) there exists an abelian subgroup $A$ of $G$ and a subgroup $A_{1}$ of $G$ such that

(a) $\Omega_{f} \widetilde{\Xi}_{G}\left(E_{1}\right)$ normalizes $A_{1}$,

(b) $A_{1} \supseteqq A \supseteqq \Omega_{f}(A)=E, E \supseteqq A_{1}$,

(c) $\Omega_{f} \mathbb{E}_{G}\left(E_{1}\right)^{\prime} \cap \mathfrak{\complement}_{G}\left(A_{1}\right) \subseteq A$,

(d) if the element $x$ of $\mathfrak{S}_{G}\left(E_{1}\right)$ is restrained by $f$, then $x$ centralizes $A_{1} / \Omega_{f}(A)$.

Then $\Omega_{f} \mathfrak{\Im}_{G}\left(E_{1}\right) \subseteq E$.

Proof. Assume that the proposition is false and choose $h \in f$ minimal with respect to $0 \leqq h \leqq f$ and $\Omega_{h} \mathfrak{\subseteq}_{G}\left(E_{1}\right) \nsubseteq E$. So by (2), $\Omega_{h} \mathfrak{\subseteq}_{G}\left(E_{1}\right)$ is not restrained by $f$.

Aiming at a contrary statement pick $x \neq 1$ and $y$ in $\mathbb{S}_{G}\left(E_{1}\right)$ where $x$ is restrained by $h$ and $y$ is restrained by $f$. We shall examine $\langle x, y\rangle$.

By $(3 d), x$ and $y$ centralize $A_{1} / \Omega_{f}(A)$. Hence $\langle x, y\rangle$ induces an abelian group of automorphisms in $A_{1}$, whence

$$
(x, y) \in \Omega_{f} \mathfrak{\Im}_{G}\left(E_{1}\right)^{\prime} \cap \mathfrak{\mho}_{G}\left(A_{1}\right) \subseteq A
$$

by (3c). But again $x$ and $y$ centralize $A / E$, so $(x, y, x)$ and $(x, y, y)$ are in $E$, and $\langle x, y\rangle$ has class of nilpotency at most 3. By Lemma 1.1,

$$
y=y^{x^{e}}=y(y, x)^{e}(y, x, x)^{\left(\begin{array}{c}
e \\
2
\end{array}\right)},
$$


hence

$$
1=(y, x)^{e}(y, x, x)^{\left(\begin{array}{c}
e \\
2
\end{array}\right)}
$$

for every natural number $e$ that is divisible by the order of $x$. Let $q$ be a prime that divides the order of $x$. Let $g \in f$ be 1 at $q$ and 0 elsewhere and let $k$ be the smallest function $\geqq 0$ in $f$ that restrains $x$. Then $x^{q}$ is restrained by $k-g$, hence $x^{q} \in \Omega_{k-g} \mathbb{E}_{G}\left(E_{1}\right)$. We have still $k-g \geqq 0$, but $k-g<k \leqq h$, since $k$ is finite. So the minimality of $h$ yields $\Omega_{k-g} \mathbb{S}_{G}\left(E_{1}\right) \leqq E \leqq A$, hence

$$
1=\left(y, x, x^{q}\right)=(y, x, x)^{q},
$$

cf. $[9$, p. $80,(9)]$.

Assume first that $h(2) \neq 1$ and let $y$ be restrained even by $h$. Choose $e$ to be the least common multiple of $q^{h(q)}$ and the orders of $x$ and $y$. Note that $h(q) \geqq d(q)$, since $q$ divides the order of $x$. Therefore $q$ divides $\left(\begin{array}{l}e \\ 2\end{array}\right)$, entailing $(y, x, x)^{\left(\begin{array}{c}e \\ 2\end{array}\right)}=1$ because of $(* 2)$ and $(y, x)^{e}=1$ because of $(* 1)$. By the choice of $e$ this proves that $(y, x)$ is contained in $\Omega_{h}(A) \leqq E$. Therefore (cf. [9, p. 80, (9)]), $(y, x)^{e}=\left(y, x^{q}\right)=$ 1, since $x^{q} \in \Omega_{k-g} \bigodot_{G}\left(E_{1}\right) \subseteq E$, and (cf. [9, p. 81, (10)])

$$
(x y)^{e}=x^{e} y^{e}(y, x)^{\left(\begin{array}{l}
e \\
2
\end{array}\right)}=1 \text {. }
$$

This proves that $\Omega_{h} \widetilde{E}_{G}\left(E_{1}\right)$ is restrained by $h \leqq f$; a contradiction.

Assume now that $h(2)=1$ and let $h^{\prime} \in \&$ have the value 2 at 2 and coincide with $h$ elsewhere. Then $h \leqq h^{\prime} \leqq f$, since $1=h(2) \leqq f(2) \neq 1$. Suppose that $y$ is restrained by $h^{\prime}$ and choose $e^{\prime}$ to be the least common multiple of $4=2^{h^{\prime}(2)}$ and the orders of $x$ and $y$. Again $q$ divides $\left(\begin{array}{l}e^{\prime} \\ 2\end{array}\right),(y, x)$ is contained in $\Omega_{h^{\prime}}(A) \leqq E$, and $(x y)^{e^{\prime}}=1$. This proves that $x y$ is restrained by $h^{\prime}$ and hence that $\Omega_{h} \mathbb{E}_{G}\left(E_{1}\right)$ is restrained by $h^{\prime} \leqq f$, if $h(2)=1$; again a contradiction.

THEOREM 2.2. If $f$ is a function in $f$, if $G$ is a hypercylic group, and if $E$ is maximal among its abelian, normal subgroups restrained by $f$, then $\Omega_{f} \mho_{G}(E)=E$, provided that $f(2) \neq 1$ and that

(*) there exists an abelian, normal torsion subgroup $A \supseteq E$ of $G$ such that $\Omega_{f} \mathfrak{\Im}_{G}(E)^{\prime} \cap \mathfrak{S}_{G}(A) \subseteq A$ and $\Omega_{d}(A)=\Omega_{d}(E)$.

Proof. We have to establish the hypotheses of Proposition 2.1. Without loss of generality $f \geqq 0$. Let $E_{1}=E$. Assume by way of contradiction that (2) is not satisfied, so that $E Q_{h} \mathfrak{F}_{G}(E) \supset E$ for some $h$ in $f$ with $0 \leqq h \leqq f$ such that $\Omega_{h} \mathbb{E}_{G}(E)$ is restrained by $f$. Then by hypercylicity, $E \Omega_{h} \mathbb{E}_{G}(E) / E$ contains a cyclic, normal subgroup $H / E \neq 1$ of $G / E$. But $E \subseteq 3(H)$, hence $H=3(H)$. Furthermore $E \Omega_{h} \mathbb{S}_{G}(E)$ is 
restrained by $f$, and so is $H$. This contradicts the maximality of $E$. Hence (2) is satisfied. Put $A_{1}=A$. By maximality of $E, \Omega_{f}(A)=E$. So $(3 a, b, c)$ are satisfied. Now let $x \in \mathfrak{C}_{G} \Omega_{f}(A)$ be restrained by $f$. Then $x$ centralizes $E$ and $\Omega_{d}(E)=\Omega_{d}(A)$, so that Theorem 1.11 is applicable, $x$ centralizes $A / \Omega_{f}(A)$. By Proposition 2.1, $\Omega_{f} \mathfrak{E}_{G}(E)=E$.

REMARK 2.3. The condition (*) in Theorem 2.2 may not be dropped as shown by the following example of [2, p. 19, Example 1]. Let $A$ be a torsionfree, abelian group and $x$ the automorphism of $A$ that sends every element onto its inverse. Then $G=A\langle x\rangle$ is hypercylic and every element in the coset $A x$ is of order 2 . Let $f=\infty$. Then $E=1$ is a maximal abelian, normal subgroup of $G$ restrained by $f$, but $\Omega_{f} \mathfrak{S}_{G}(E)=G \neq E$.

REMaRK 2.4. As already indicated by J. L. Alperin the condition $f(2) \neq 1$ in Theorem 2.2 may not be dropped. If $G$ is a dihedral group of order $2^{n+1} \geqq 16$, then $G$ has no elementary abelian, normal subgroups of index 2 , since $G$ contains elements of order 8 . Therefore it follows from [8, Lemma 1] that a maximal elementary abelian, normal subgroup $E$ of $G$ has order 2 and as such lies in the center of $G$. But at least half of the elements of $G$ have order 2. This proves $\Omega_{1} \Subset_{G}(E) \supset E$. This question has been investigated further by G. Tani Corsi [7].

CoRollary 2.5. If $f \geqq 0$ is a function in $f$, if $G$ is a hypercylic group, and if $E$ is maximal among its abelian, normal subgroups restrained by $f$, then $\Omega_{f} \Im_{G}(E)=E$ provided that one of the following holds:

(1) $f(2) \neq 1$ and $\Omega_{f}(G)$ is restrained by $\bar{f}$ where $\bar{f}(p)=0$ if $f(p) \leqq 0$ and $\bar{f}(p)=\infty$ if $f(p)>0$.

(2) $f \geqq d$ and $\Omega_{f}(G)$ is a torsion group.

(3) There exists a prime $q$ such that $f(p)=0$ for $p \leqq q$ and $f(p) \geqq 1$ for $p>q$.

(4) $f(2) \neq 1$ and the set of p-elements of $G$ is a subgroup for every prime $p$.

(5) $f(2) \neq 1$ and $G$ is hypercentral.

Proof. Assume (1). It will suffice to show that condition $\left(^{*}\right)$ of Theorem 2.2 is satisfied. Let $A$ be maximal among the abelian, normal subgroups of $G$ containing $E$ and restrained by $\bar{f}$; such a subgroup exists by the maximum principle of set theory. $F=\Omega_{f}(G) \cap \mathfrak{V}_{G}(A)$ is restrained by $\bar{f}$. Therefore, since $G$ is hypercylic, a similar argument as used in the proof of Theorem 2.2 shows that $F$ is contained in $A$. In particular, $\Omega_{f}(G)^{\prime} \cap \mathfrak{E}_{G}(A) \subseteq F \leqq A$. If for some prime $p$ the component $A_{p}$ of $A$ is not 1 , then $\bar{f}(p)=\infty$ and $f(p)>0$, hence 
$f(p) \geqq d(p)$. Therefore, $\Omega_{d}(A) \subseteq \Omega_{f}(A)$. By the maximality of $E$, $\Omega_{f}(A)=E$. This implies $\Omega_{d}(A)=\Omega_{d}(E)$, and Theorem 2.2 is applicable.

(2) is a special case of (1).

In any hypercylic group $G$ the torsion elements of an order divisible by primes $p>q$ only form a subgroup $G(q)$; cf. [2, p. 21, Proposition 1]. Therefore (3) is also a special case of (1).

Clearly (4) implies (1).

Every hypercentral group is locally nilpotent [5, p. 223] and every locally nilpotent group has a unique $p$-Sylow subgroup for every prime $p[5, \mathrm{p} .229]$. Hence (5) is a special case of (4).

Corollary 2.6. For a p-Sylow subgroup $P$ of a finite group $G$ let $E$ be maximal among the abelian, normal subgroups of $P$ of exponent dividing $p^{n}, n \geqq d(p)$. Then $\mathfrak{S}_{G}(E)$ has a normal p-complement and $E$ is the set of all elements in $\mathfrak{E}_{G}(E)$ of order dividing $p^{n}$.

Proof. Since $P$ normalizes $C=\mathfrak{\Im}_{G}(E), S=\mathfrak{\Im}_{P}(E)$ is a $p$-Sylow subgroup of $C$. Moreover, $\Omega_{d}(S)=\Omega_{d} \mathbb{C}_{P}(E) \subseteq E$ by Corollary 2.5 (5), hence Corollary 1.5 yields the existence of a normal $p$-complement in $C$. An arbitrary $p$-Sylow subgroup $S_{0}$ of $C$ is conjugate to $S$ in $C=$ $\mathfrak{G}_{G}(E)$. Therefore $E=\Omega_{n}(S)=\Omega_{n}\left(S_{0}\right)$. This completes the proof.

Definition 2.7. (a) For $f \in f$ define $f^{\prime} \in f$ by $f^{\prime}(p)=0$ if $f(p) \leqq 0$ and $f^{\prime}(p)=1$ if $f(p)>0$.

(b) For an abelian normal subgroup $E$ of a group $G$ such that $E$ is restrained by $f \in f$ define $\mathfrak{X}_{G}^{f}(E)$ by

$$
\mathfrak{X}_{G}^{f}(E) / E=\Omega_{f}\left[\Omega_{f} \mathfrak{S}_{G}(E) / E \cap \Re(G / E)\right] \text {. }
$$

THEOREM 2.8. If $f$ is a function in $f$ with $f(2) \neq 1$, if $G$ is a hypercentral group, and if $E$ is maximal among its abelian characteristic subgroups restrained by $f$, then $\Omega_{f} \mathfrak{S}_{G} \mathfrak{X}_{G}^{f}(E)=E$.

Proof. (1) Let $U$ be a normal subgroup of $G$ contained in $\mathfrak{C}_{G} \mathfrak{X}_{G}^{f}(E)$ and restrained by $f$, and assume by way of contradiction that $U \nsubseteq E$. Then $U E / E \neq 1$, whence $U E / E \cap 3(G / E) \neq 1$ by hypercentrality. Since $U E$ is restrained by $f$ we see that $\Omega_{1}(S)=\Omega_{f^{\prime}}(S)$ for every subgroup $S$ of $U E / E$, in particular

$$
1 \neq \Omega_{f^{\prime}}[U E / E \cap 3(G / E)] \subseteq\left[U E \cap \mathfrak{X}_{G}^{f}(E)\right] / E .
$$

But $U E \cap \mathfrak{X}_{G}^{f}(E)=\left[U \cap \mathfrak{X}_{G}^{f}(E)\right] E$, where $U \cap \mathfrak{X}_{G}^{f}(E) \subseteq \Omega_{f} \mathfrak{X}_{G}^{f}(E) \subseteq E$ by the maximality of $E$. This contradiction shows that $U \subseteq E$.

(2) Consider first the case where $f$ assumes only the values 0 and $\infty$. Since $G$ is hypercentral, $\Omega_{f}(G)$ is restrained by $f$ (cf. proof 
of Corollary $2.5(5))$ and so is $U=\Omega_{f} \mathbb{C}_{G} \mathfrak{x}_{G}^{f}(E)$. Therefore (1) implies $\Omega_{f} \mathfrak{E}_{G} \mathfrak{X}_{G}^{f}(E) \subseteq E$. Clearly $E \subseteq \Omega_{f} \mathfrak{E}_{G} \mathfrak{X}_{G}^{f}(E)$, and the theorem is proved in this case.

(3) Now consider the general case and put $E_{1}=\mathfrak{X}_{G}^{f}(E)$. Then condition (2) of Proposition 2.1 follows from (1) above. Let $A$ be maximal among the abelian, characteristic subgroups of $G$ containing $E$ and restrained by $\bar{f}$, where $\bar{f}$ is defined as in Corollary 2.5 (1). Put $A_{1}=\mathfrak{X}_{G}^{\bar{f}}(A)$. Then $\Omega_{f}(A)=E$ by the maximality of $E$ and $\Omega_{\bar{f}} \mathfrak{S}_{G} \mathfrak{X}_{G}^{\bar{f}}(A)=$ $A$ by (2) above. In particular since $\Omega_{f} \overleftarrow{E}_{G}\left(E_{1}\right)^{\prime} \subseteq \Omega_{\bar{f}}(G)$ is restrained by $\bar{f}$,

$$
\Omega_{f} \mathfrak{S}_{G}\left(E_{1}\right)^{\prime} \cap \mathfrak{C}_{G}\left(A_{1}\right) \subseteq \Omega_{\bar{f}} \mathfrak{S}_{G}\left(A_{1}\right)=A
$$

proving (3c) of Proposition 2.1. Clearly $A_{1} / A$ is centralized by every element in $G$. As in the proof of Corollary 2.5 $(1), \Omega_{d}(A)=\Omega_{d}(E)$. Therefore (3d) follows from Theorem 1.11, and Proposition 2.1 yields $\Omega_{f} \mathfrak{s}_{G}\left(E_{1}\right)=E$.

Corollary 2.9. For a p-Sylow subgroup $P$ of a finite group $G$ let $E$ be maximal among the abelian, characteristic subgroups of $P$ of exponent dividing $p^{n}, n \geqq d(p)$. Then $\mathfrak{}_{G} \mathfrak{X}_{P}^{n}(E)$ has a normal $p$ complement and $E$ is the set of all elements in $\mathfrak{S}_{G} \mathfrak{X}_{P}^{n}(E)$ of order dividing $p^{n}$.

Proof. Use Theorem 2.8 in the proof of Corollary 2.6.

\section{REFERENCES}

1. J. L. Alperin, Centralizers of abelian normal subgroups of p-groups, J. Algebra 1 (1964), 110-113.

2. R. Baer, Supersoluble groups, Proc. Amer. Math. Soc. 6 (1955), 16-32.

3. G. Frobenius, Über auflösbare Gruppen, V, Sitzungsberichte preuß. Akad. Wiss. Berlin (1901), 1324-1329.

4. B. Huppert, Subnormale Untergruppen und p-Sylowgruppen, Acta Sci. Math. Szeged 22 (1961), 46-61.

5. A. G. Kurosh, The Theory of groups, II, second English edition, Chelsea Publ. Co. New York, N. Y.

6. H. Leptin, Einige Bemerkungen ïber die Automorphismen abelscher p-Gruppen, Proc. Colloq. Abelian Groups Tihany 1963; Budapest (1964), 99-104.

7. G. Tani Corsi, Sui centralizzanti dei sottogruppi normali abeliani de 2-gruppi, Le Matematiche (Catania) 20 (1965), 137-141.

8. J. G. Thompson, A special class of non-solvable groups, Math. Z. 72 (1960), 458-462.

9. H. J. Zassenhaus, The theory of groups, second edition, Chelsea Publ. Co. New York, N.Y.

Received November 18, 1968.

New Mexico State University, Las Cruces

UnIVERSity of Missouri, St. Louis 


\section{PACIFIC JOURNAL OF MATHEMATICS}

\section{EDITORS}

H. ROYDEN

Stanford University

Stanford, California

Richard Pierce

University of Washington

Seattle, Washington 98105
J. DUGUNDJI

Department of Mathematics

University of Southern California

Los Angeles, California 90007

BASIL GORDON

University of California

Los Angeles, California 90024

\section{ASSOCIATE EDITORS}
E. F. BECKENBACH
B. H. NEUMANN
F. WOLF
K. YosHIDA

\section{SUPPORTING INSTITUTIONS}

UNIVERSITY OF BRITISH COLUMBIA

CALIFORNIA INSTITUTE OF TECHNOLOGY

UNIVERSITY OF CALIFORNIA

MONTANA STATE UNIVERSITY

UNIVERSITY OF NEVADA

NEW MEXICO STATE UNIVERSITY

OREGON STATE UNIVERSITY

UNIVERSITY OF OREGON

OSAKA UNIVERSITY

UNIVERSITY OF SOUTHERN CALIFORNIA
STANFORD UNIVERSITY

UNIVERSITY OF TOKYO

UNIVERSITY OF UTAH

WASHINGTON STATE UNIVERSITY

UNIVERSITY OF WASHINGTON

*

AMERICAN MATHEMATICAL SOCIETY CHEVRON RESEARCH CORPORATION TRW SYSTEMS

NAVAL WEAPONS CENTER 


\section{Pacific Journal of Mathematics}

\section{Vol. 31, No. $1 \quad$ November, 1969}

James Burton Ax, Injective endomorphisms of varieties and schemes........

Richard Hindman Bouldin, A generalization of the Weinstein-Aronszajn

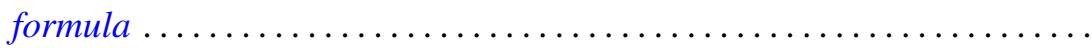

John Martin Chadam, The asymptotic behavior of the Klein-Gordon equation with external potential. II ...............................

Rina Hadass, On the zeros of the solutions of the differential equation

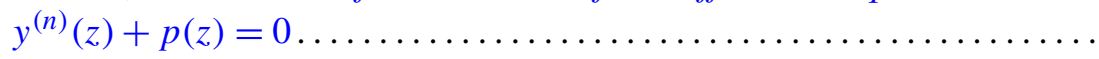

John Sollion Hsia, Integral equivalence of vectors over local modular lattices. II .............................................

Robert Hughes, Boundary behavior of random valued heat polynomial

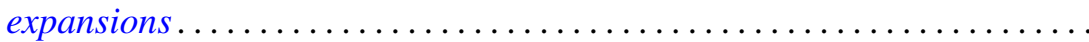

Surender Kumar Jain, Saad H. Mohamed and Surjeet Singh, Rings in which every right ideal is quasi-injective .........................

T. Kawata, On the inversion formula for the characteristic function .........

Erwin Kleinfeld, On right alternative rings without proper right ideals......

Robert Leroy Kruse and David Thomas Price, On the subring structure of

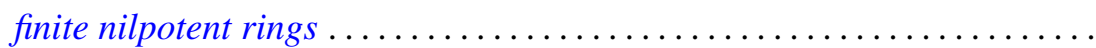

Marvin David Marcus and Stephen J. Pierce, Symmetric positive definite multilinear functionals with a given automorphism. .

William Schumacher Massey, Pontryagin squares in the Thom space of a bundle...

William Schumacher Massey, Proof of a conjecture of Whitney ...

John William Neuberger, Existence of a spectrum for nonlinear transformations

Stephen E. Newman, Measure algebras on idempotent semigroups ...

$\mathrm{K}$. Chandrasekhara Rao, Matrix transformations of some sequence spaces

Robert Bruce Schneider, Some theorems in Fourier analysis on symmetric

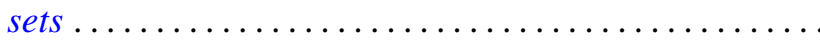

Ulrich F. K. Schoenwaelder, Centralizers of abelian, normal subgroups of hypercyclic groups...

Jerrold Norman Siegel, $G$-spaces, $H$-spaces and $W$-spaces

Robert Irving Soare, Cohesive sets and recursively enumerable Dedekind cuts...

Kwok-Wai Tam, Isometries of certain function spaces .... . .

Awadhesh Kumar Tiwary, Injective hulls of semi-simple modules over regular rings ....

Eldon Jon Vought, Concerning continua not separated by any nonaposyndetic subcontinuum .................... 